\title{
ÍNDICE DE CONSERVAÇÃO EM ÁREAS PROTEGIDAS E \\ ICMS ECOLÓGICO EM MINAS GERAIS
}

\author{
Marianne Silva Oliveira* \\ Ricardo Alexandrino Garcia
}

\section{Resumo}

O ICMS Ecológico consiste na proporção do imposto repassado aos municípios em função do critério Meio Ambiente. Em Minas Gerais, com a aprovação da lei estadual 12.040 /1995 (a "Lei Robin Hood"), o critério ambiental foi estabelecido, correspondente a 1\% do total repassado aos municípios. O Índice de Conservação é critério utilizado para o cálculo do ICMS ecológico relativo à presença de unidades de conservação nos municípios, considerando a razão entre área da UC e área municipal, o Fator de Conservação relativo à categoria de UCs e Fator de Qualidade. A variação do Índice de Conservação pode ocorrer em função do Fator de Qualidade, que é estabelecido com base na avaliação anual das áreas protegidas referente a critérios de estrutura, gestão e manejo. O estudo tem como objetivo analisar o impacto de variações do Índice de Conservação (IC) na transferência de valores do ICMS ecológico aos municípios no Estado de Minas Gerais, através de uma análise comparativa entre valores de transferência para o atual cenário e o cenário hipotético em que o Fator de Qualidade é unitário para todas categorias.

Palavras-chave: ICMS Ecológico, Áreas Protegidas, Índice de Conservação, Fator de Qualidade.

*Mestranda em Modelagem de Sistemas Ambientais/UFMG.

•Prof. Adjunto do Departamento Geografia Instituto de Geociências/UFMG. 


\section{1- INTRODUÇÃO}

O Imposto sobre Circulação de Mercadorias e Serviços (ICMS) é uma das principais fontes de receita tributária de estados e municípios, sendo gerado nas operações relativas à circulação de mercadorias, prestação de serviços, energia e comunicações. O repasse dos recursos arrecadados é regulamentado pelo artigo 158 da Constituição Federal (BRASIL, 1988) determinando que $25 \%$ do total arrecadado seja destinado aos municípios e que, desse total, no mínimo $75 \%$ sejam distribuídos segundo o valor adicionado fiscal ${ }^{1}$. Os $25 \%$ restantes devem ser distribuídos segundo critérios regulamentados pela legislação estadual.

O ICMS Ecológico consiste na proporção do imposto repassado aos municípios em função do critério Meio Ambiente. Logo, a possibilidade do estado criar o ICMS Ecológico dá-se mediante a consideração do critério ambiental no cálculo da participação de cada um dos municípios na repartição dos valores arrecadados (LOUREIRO, 2002).

No Brasil, o primeiro estado a adotar o ICMS Ecológico foi o Paraná, em 1991, como forma de "compensação" às áreas de restrições legais a expansão de atividades econômicas. O pioneirismo do Paraná foi replicado em outros estados da Federação, cada qual vinculando critérios de repasse que melhor atendessem aos interesses da população local e suas peculiaridades tais como: existência de unidades de conservação, áreas de manancial para abastecimento público, saneamento ambiental, coleta seletiva de lixo, preservação de patrimônio histórico e reservas indígenas (RIBEIRO, 2008).

Atualmente são 14 estados que possuem ICMS ecológico (TNC, 2010) com seus respectivos anos de criação: Paraná (1991); São Paulo (1993); Minas Gerais (1995); Amapá(1996); Rondônia (1996); Rio Grande do Sul (1997); Mato Grosso (2000); Mato Grosso do Sul (2000); Pernambuco (2000); Tocantins (2002); Acre (2004); Goiás (2007), Rio de Janeiro(2007) e Ceará (2008).

Até 1995 o Estado de Minas Gerais repassava o ICMS segundo dois únicos critérios: valor adicionado fiscal - VAF (94,4\%) e produção minerária (5,6\%). A partir da aprovação da Lei n¹2.040 em 28 de dezembro de 1995 (MINAS GERAIS, 1995), conhecida como "Lei Robin Hood", foram redefinidos os critérios de distribuição do ICMS, incluindo parâmetros relacionados a saúde, educação, produção de alimentos, patrimônio histórico, população e meio ambiente (MINAS GERAIS, 2005a).

De acordo com a Lei Estadual n. ${ }^{\circ}$ 13.803/2000 (MINAS GERAIS, 2000), o critério Meio Ambiente deve corresponder a $1 \%$ do total do ICMS destinado aos municípios, sendo a

\footnotetext{
${ }^{1}$ Valor Adicionado Fiscal mede a produção econômica do município trazida pela diferença entre o somatória das notas fiscais de venda e o somatório das notas fiscais de compra.
} 
distribuição deste montante realizada em função do Índice de Meio Ambiente (IMA) que consiste no somatório de 3 subcritérios ponderados pelos respectivos pesos: o Índice de Conservação (IC)-45,45\%, referente às unidades de conservação e outras áreas protegidas; o Índice de Saneamento Ambiental (ISA)-45,45\%, referente a aterros sanitários, estações de tratamento de esgotos e usinas de compostagem e, por fim, o Índice de Mata Seca (IMS)-9,1\%, este último incluído através da Lei Estadual n. ${ }^{\circ}$ 18.030/2009 (MINAS GERAIS, 2009), referente à presença e proporção em área da fitofisionomia Mata Seca.

Com foco nas unidades de conservação, o presente estudo tem como objetivo analisar o impacto de variações do Índice de Conservação (IC) na transferência de valores do ICMS ecológico aos municípios no Estado de Minas Gerais.

\section{Cálculo do Índice de Conservação}

O Índice de Conservação (IC) se refere ao município, sendo calculado a partir do Fator de Conservação Municipal (FCM) em relação ao somatório $\sum$ FCMde todos os municípios, onde $\mathrm{IC}=\underline{\mathrm{FCM}}$.

$\sum \mathrm{FCM}$

O FCM é calculado a partir da soma dos Fatores de Conservação referente a cada unidade de conservação (FCMi) presente no município. O cálculo do FCMi leva em consideração a proporção entre área da UC e área municipal, o Fator de Conservação (FC) e o Fator de Qualidade (FQ) de acordo com a fórmula:

$\mathrm{FCMi}=\underline{\text { área } \mathrm{UC}} \cdot \mathrm{FC} . \mathrm{FQ}$

área município

O Fator de Conservação (FC) é um valor fixo que varia de 0,025 a 1 de acordo com a categoria da UC, estabelecidos pela Resolução SEMAD n. 318 de 15 de fevereiro de 2005 (MINAS GERAIS, 2005b) e que foram alterados pela Lei n. ${ }^{\circ}$ 18.030/2009 (MINAS GERAIS, 2009) (Tabela 1), sendo estes últimos valores fixados utilizados no cálculo do ICMS ecológico para o ano de 2010.

O Fator de Qualidade (FQ) pode variar de 0,1 a 1 sendo estabelecido com base na avaliação anual das unidades de conservação referente a estrutura, gestão e manejo, cujos procedimentos, critérios e normas estão definidos pela Deliberação Normativa COPAM no 86 de 17 de julho de 2005 (MINAS GERAIS, 2005c). 
Tabela1: Fator de Conservação por categoria de Unidades de Conservação segundo Resolução SEMAD 2005 e Lei Estadual 18.030/2009

\begin{tabular}{|c|c|c|c|c|}
\hline Grupo & Categori & & FC SEMAD 2005 & FC LEI 2009 \\
\hline \multirow{5}{*}{$\begin{array}{l}\text { Proteção } \\
\text { Integral }\end{array}$} & EE & Estação Ecológica & 1,0 & 1,0 \\
\hline & $\mathrm{RB}$ & Reserva Biológica & 1,0 & 1,0 \\
\hline & MNA & Monumento Natural & 1,0 & 1,0 \\
\hline & RVS & Refúgio de Vida Silvestre & 1,0 & 1,0 \\
\hline & PAQ & Parque & 0,9 & 1,0 \\
\hline \multirow{9}{*}{$\begin{array}{l}\text { Uso } \\
\text { Sustentável }\end{array}$} & RESEX & Reserva Extrativista & 0,5 & 0,5 \\
\hline & RDS & Reserva de Desenvolvimento Sustentável & 0,7 & 0,5 \\
\hline & FLO & Floresta & 0,7 & 0,3 \\
\hline & RF & Reserva de Fauna & 0,3 & 0,3 \\
\hline & ARIE & Área de Relevante Interesse Ecológico & 0,3 & 0,3 \\
\hline & APA & $\begin{array}{l}\text { Área de Proteção Ambiental } \\
\text { com zoneamento: Zona de vida Silvestre }\end{array}$ & 1,0 & 0,5 \\
\hline & APA & $\begin{array}{l}\text { Área de Proteção Ambiental, com } \\
\text { zoneamento: Demais zonas }\end{array}$ & 0,1 & 0,1 \\
\hline & APA & $\begin{array}{l}\text { Área de Proteção Ambiental, sem } \\
\text { zoneamento }\end{array}$ & 0,025 & 0,025 \\
\hline & RPPN & Reserva Particular do Patrimônio Natural & 0,9 & 1,0 \\
\hline \multirow[t]{3}{*}{$\begin{array}{l}\text { Outros } \\
\text { grupos }\end{array}$} & RPRA & $\begin{array}{l}\text { Reserva Particular de Recomposição } \\
\text { Ambiental }\end{array}$ & 0,9 & 0,9 \\
\hline & Al & Área Indígena & 0,5 & 0,5 \\
\hline & APE & Áreas de Proteção Especial Estaduais & 0,1 & 0,1 \\
\hline
\end{tabular}

Embora regulamentado desde 2005, o Fator de Qualidade foi aplicado como valor unitário para todas as categorias de UCs até o ano de 2009, quando foi então realizada a avaliação anual de UCs pelo órgão ambiental, estabelecendo os valores para o índice FQ que estão sendo aplicados no cálculo do ICMS Ecológico para o ano de 2010.

Compreendidos os parâmetros utilizados no cálculo do Índice de Conservação dos municípios, é possível estimar a variação do IC a partir de alterações no Fator de Qualidade, visto que o Fator de Conservação é um critério fixo e considerando constante a razão entre área de UC e área municipal.

\section{Quadro Atual de UCs e transferências de ICMS Ecológico}

O valor das transferências do ICMS ecológico proporcional às unidades de conservação e aos municípios são disponibilizados pela Fundação João Pinheiro (FJP, 2010) para períodos mensais e anuais, além das informações referentes ao índice de conservação de municípios e UCs, incluindo os respectivos Fatores de Conservação e de Qualidade. 
Com base nos dados do repasse de ICMS Ecológico para o mês de outubro de 2010, o total transferido foi de aproximadamente 2 milhões de reais referente ao subcritério conservação, contemplando 342 municípios (Figura 1A).
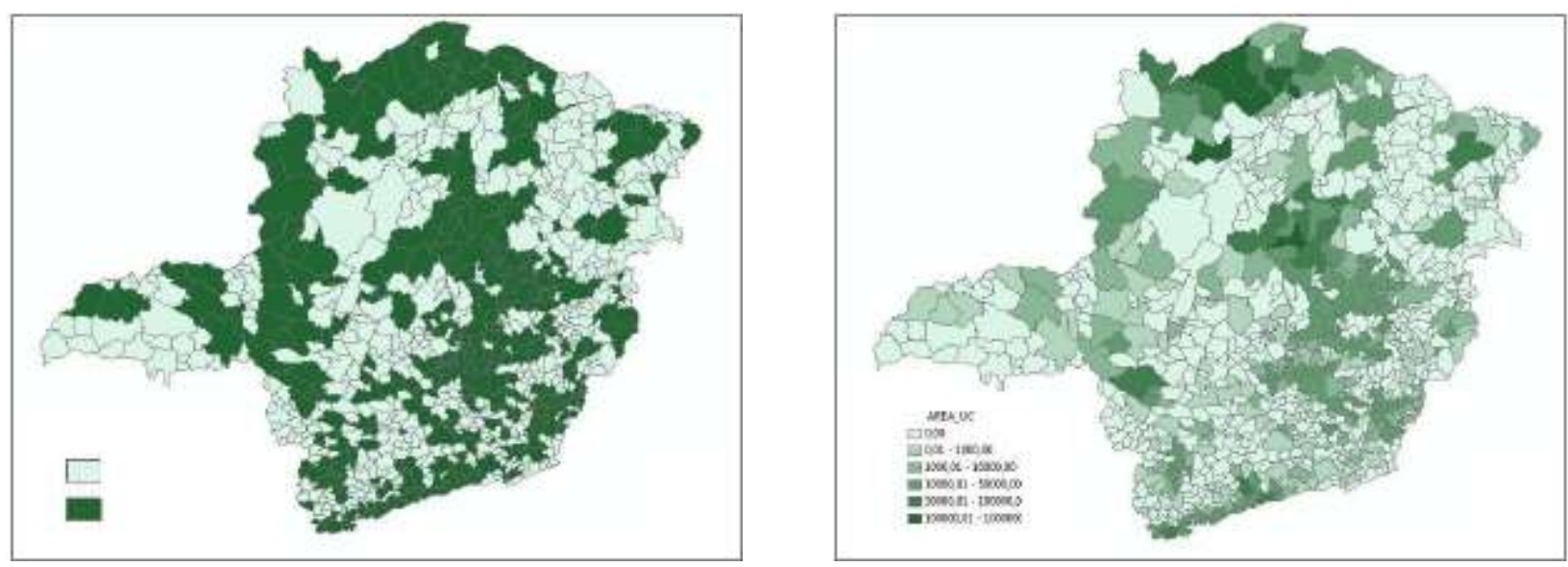

Figura 1: (A) Municípios com ICMS Ecológico subcritério conservação e (B) Área de UCs em hectares por município no Estado de Minas Gerais.

O total de 497 unidades de conservação ocupam aproximadamente 5 milhões de hectares (Tabela 2), equivalentes a 8,4\%da área total do estado, com distribuição heterogêneaentre municípios (Figura 1B).

Tabela 2: Unidades de Conservação - Categorias Proteção Integral e Uso Sustentável

\begin{tabular}{|r|r|r|c|r|}
\hline categoria UC & \multicolumn{1}{c|}{ Quantidade } & \multicolumn{1}{c|}{ Área (ha) } & Indice de Conservaçäo & \multicolumn{1}{c}{ Total RS } \\
\hline Integral & 101 & $1.050 .153,37$ & 4.68690668 & $821.024,65$ \\
\hline Sustentável & 396 & $3.880 .287,81$ & 7,30163375 & $1.266 .584,75$ \\
\hline TOTAL MG & 497 & $4.930 .441,18$ & 11,96854041 & $\mathbf{2 . 0 8 7 . 6 0 9 , 4 1}$ \\
\hline \hline
\end{tabular}
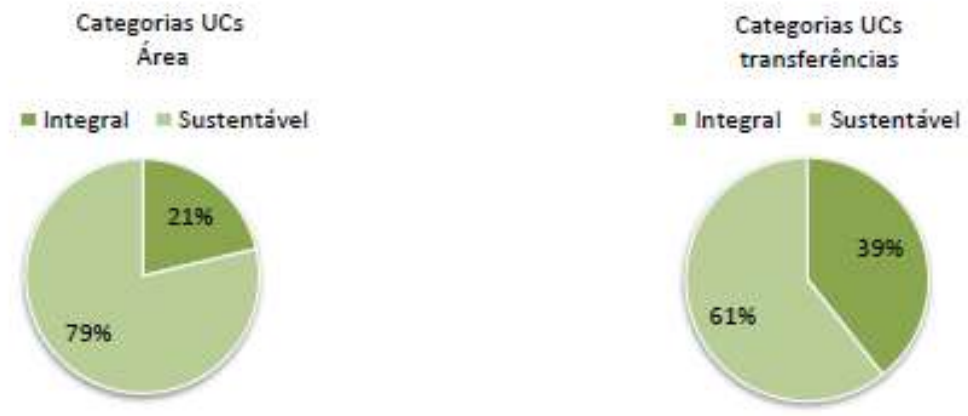

Figura 2: Área e transferência de ICMS Ecológico nas diferentes categorias de Unidades de Conservação. 
Com relação às diferentes categorias, áreas de Uso Sustentável são aproximadamente quatro vezes maiores em quantidade e extensão do que áreas de proteção integral, sendo estas responsáveis pela transferência de mais 61\% dos recursos (Figura 2).

Com relação ao âmbito da administração, as áreas protegidas estaduais correspondem a $47 \%$ do total de transferências, seguida pelo âmbito municipal em 30\% e sendo o âmbito federal de menor proporção, $23 \%$. Os valores de transferências são aproximados ao total correspondente em área nos diferentes âmbitos (Figura 3).
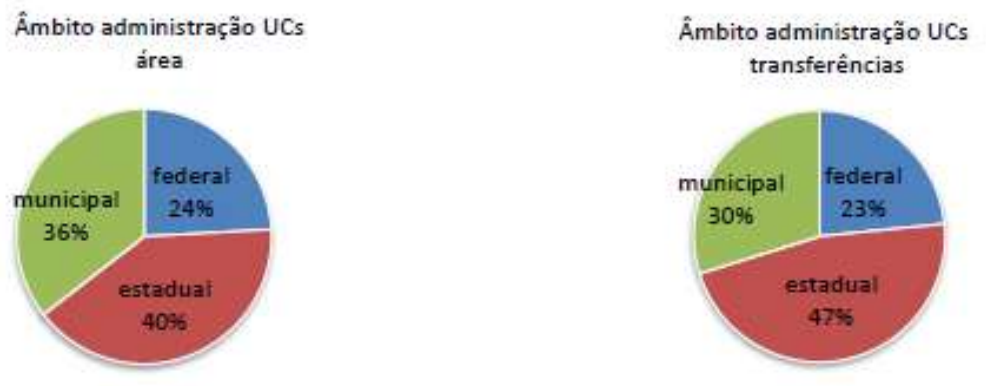

Figura 3: Arrecadação de Unidades de Conservação nos âmbitos da administração.

As unidades de proteção integral são majoritariamente representadas pela categoria Parques (PAQ) que correspondem a mais de 90\% da área e do total transferido. Em seguida, os Refúgios de Vida Silvestre (RVS) apresentam o maior índice de conservação, o que garante valores de transferências maiores do que a soma dos valores repassados às Reservas Biológicas (RB) e as Estações Ecológicas (EE), estas que juntas representam uma área 6 vezes mais extensa do que a categoria RVS (Tabela 3).

Tabela 3: Unidades de Conservação - Categoria Proteção Integral

\begin{tabular}{|c|r|r|r|r|}
\hline Categoria UC & Quantidade & \multicolumn{1}{c|}{ Área (ha) } & indice de Conservação & \multicolumn{1}{c|}{ Total RS } \\
\hline EE & 13 & 13.496 .23 & 0,07063466 & $12.395,87$ \\
\hline RB & 9 & $63.684,09$ & 0,04522517 & $8.010,02$ \\
\hline PAQ & 69 & 956.887 .17 & 4.38357068 & 771.163 .80 \\
\hline RVS & 3 & $12.542,36$ & 0,16246918 & $28.580,13$ \\
\hline MNA & 7 & 3.543 .53 & 0,00500697 & 874.83 \\
\hline total & 101 & $1.050 .153,37$ & 4,66690666 & 821.024 .65 \\
\hline
\end{tabular}

Esta disparidade pode estar relacionada à atribuição do Fator de Qualidade (FQ) unitário para RVS, enquanto as categorias EE e RB com variação de FQ no intervalo de 0,10 a 0,8 resultando em menores índices de conservação. Os Monumentos Naturais (MNA) tem representatividade minoritária em área e valores de transferências. 
Com relação às unidades de Uso Sustentável, as Áreas de Proteção Ambiental (APA) são as representantes majoritárias em área correspondendo a aproximadamente $70 \%$ dos valores transferidos. A categoria RPPN - Reserva Particular do Patrimônio Natural ocorre em maior quantidade e corresponde a $16 \%$ das transferências, o segundo maior valor (Tabela 4). As Categorias Florestas (FLO) e a Reserva do Desenvolvimento Sustentável (RDS) tem representação minoritária em quantidade e extensão. As Áreas de Proteção Especial (APE) e Áreas Indígenas (AI), respectivamente afiliadas aos âmbitos estadual e federal, apresentam proximidade entre valores de transferência. Embora a APE tenha extensão 3 vezes maior do que AI, ambas apresentam índice de conservação aproximado, em função do Fator de Conservação (FC) fixado respectivamente para 0,1 e 0,5.

Tabela 4: Unidades de Conservação - Categoria Uso Sustentável

\begin{tabular}{|c|r|r|r|r|}
\hline Categoria UC & Quantidade & Área (ha) & índice de Conservação & \multicolumn{1}{c|}{ Total R\$ } \\
\hline APE & 20 & $197.461,18$ & 0,54621481 & $96.085,23$ \\
\hline Al & 4 & $59.359,00$ & 0,48743132 & $85.744,57$ \\
\hline APA & 140 & $3.443 .813,80$ & 5,11174571 & $881.510,13$ \\
\hline FLO & 5 & $5.163,37$ & 0,00805890 & 1.417 .65 \\
\hline RDS & 1 & $60.820,33$ & 0,01963398 & $3.453,83$ \\
\hline RPPN & 226 & $113.570,13$ & 1,12854904 & $198.373,34$ \\
\hline total & 396 & $3.880 .287,81$ & 7,30163375 & $1.266 .584,75$ \\
\hline
\end{tabular}

\section{Variação do IC Fator de Qualidade}

Com objetivo de analisar o impacto da variação do Índice de Conservação nas transferências de ICMS Ecológico, foi realizada uma análise comparativa entre o cenário atual e o cenário hipotético em que o Fator de Qualidade seja unitário para todas as categorias. A estimativa será realizada a partir dos dados básicos referentes ao repasse de ICMS para o mês de Setembro de 2010.

Para o cenário FQ unitário, o somatório dos Fatores de Conservação Municipal (FCM) que se refere ao total do estado varia de 10,62 para 25,57 reduzindo por consequência os valores dos IC em relação ao cenário atual. Essa variação será mais evidente para categorias com baixos valores de FCM, associados a menores valores de razão entre áreas de UC e área municipal. A variação das transferências aos municípios se concentrou nas faixas de valores de 1 a 15 mil reais (Figura 4). 

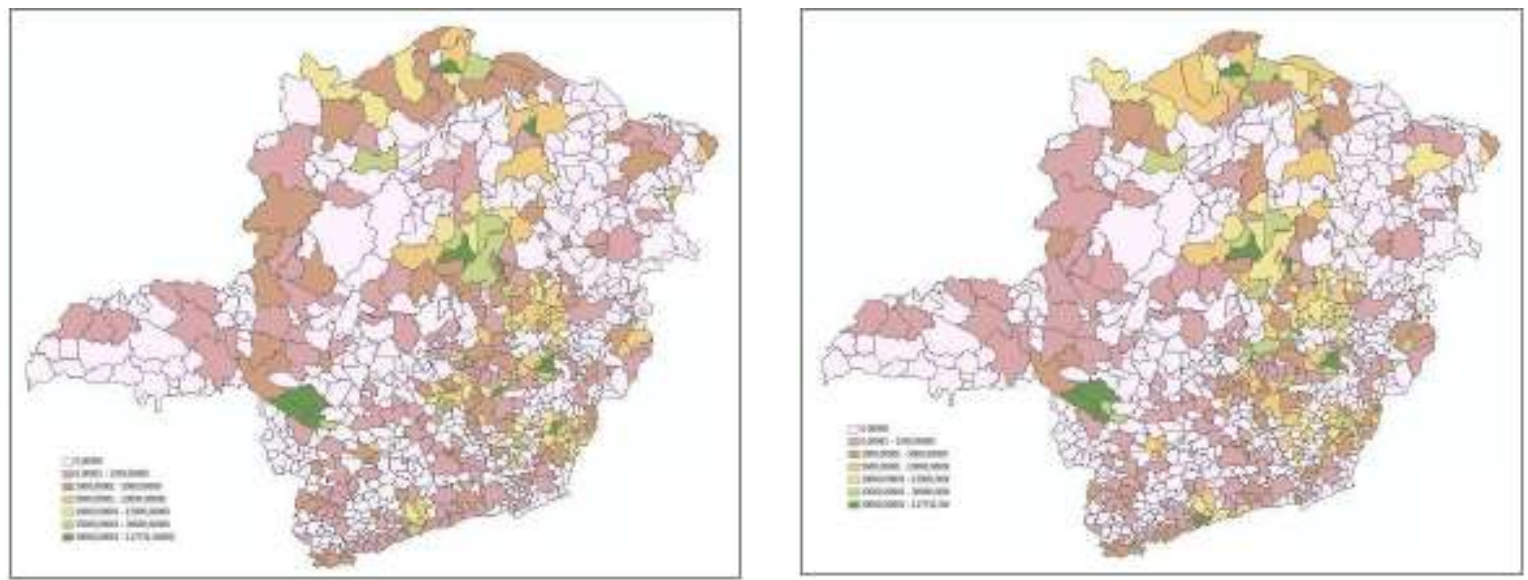

Figura 4: Total de transferências por municípios para o cenário hipotético com FQ unitário (A) e cenário atual (B).

Nas unidades de Proteção Integral, com exceção dos Parques Estaduais, todas as demais categorias foram desfavorecidas, apresentando menores valores de transferência para o cenário atual em relação ao cenário hipotético (Figura 5). A soma das diferenças entre cenários para as categorias totaliza aproximadamente 60 mil reais que deixaram de ser transferidos. Os Parques Estaduais, única categoria favorecida no cenário atual, apresentou acréscimo de 100 mil reais nos recursos transferidos.
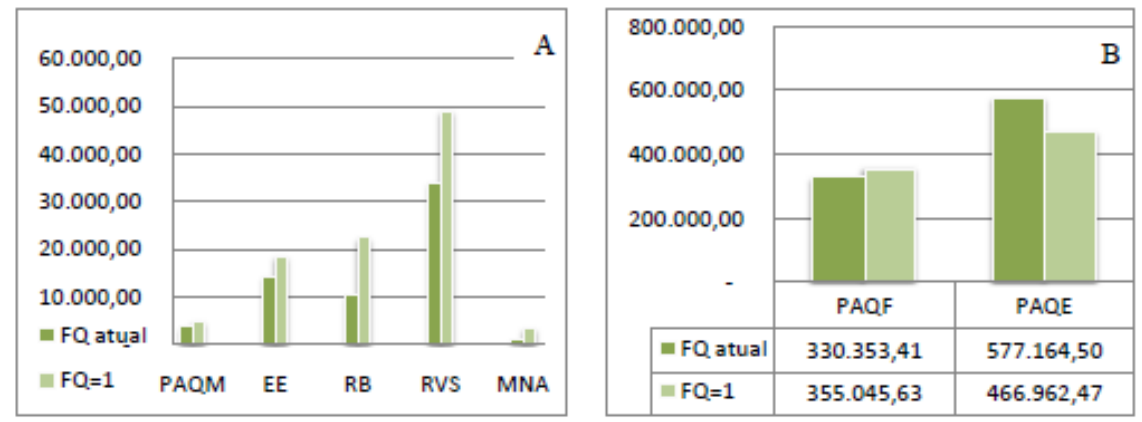

Figura 5: Análise Comparativa de UCs de Proteção Integral para Fator de Qualidade (FQ) atual e unitário.

Para as unidades de Uso Sustentável, grande parte das categorias foi favorecida no cenário atual, com destaque para APE, AI e RPPN divida nos âmbito federal e estadual, apresentando acréscimos médios de aproximadamente 65 mil reais em transferências (Figura 6). A categoria APA foi a mais desfavorecida no cenário atual, com decréscimo de mais de $70 \%$ nas transferências, com destaque para as APA municipais, responsáveis pelos maiores valores destinados à categoria. 


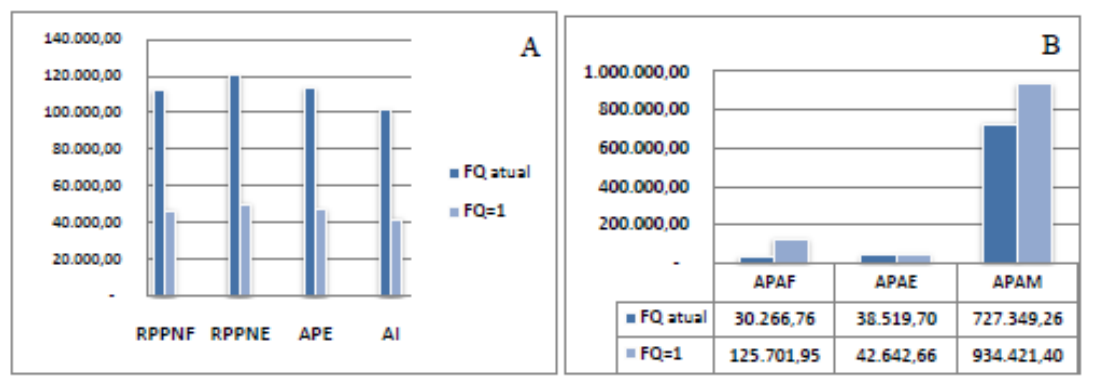

Figura 6: Análise comparativa de UCs de Uso Sustentável para Fator de Qualidade (FQ) atual e unitário.

\section{Considerações Finais}

A aplicação do Fator de Qualidade unitário para todas as categorias de UCs favorece municípios com maiores áreas e associadas a Fatores de Conservação mais altos, no caso áreas de proteção integral. No cenário atual, o Fator de Qualidade atenua a transferência majoritária para a categoria das APAs, em função da sua grande extensão, redistribuindo os recursos de forma mais igualitária para outras categorias de uso sustentável. Diante deste quadro, a aplicação dos valores atuais de FQ devem ser a investigados no que se refere a possíveis variações que possam refletir a melhoria dos critérios para distribuição dos recursos do ICMS Ecológico.

\section{REFERÊNCIAS BIBLIOGRÁFICAS}

BRASIL. Constituição (1988). Constituição da República Federativa do Brasil,1988. Brasília: Senado Federal; Centro Gráfico, 1988.

FJP - FUNDAÇÃO JOÃO PINHEIRO. Lei Robin Hood. Belo Horizonte: FJP, 2003.Disponível em: <http://www.fjp.gov.br/produtos/cees/robin_hood>. Acesso em: 12 nov. 2010.

LOUREIRO, W. ICMS ecológico: a consolidação de uma experiência brasileira de incentivo à conservação da biodiversidade. In:CONGRESSO BRASILEIRO DE UNIDADES DE CONSERVAÇÃO, 3., 2002, Fortaleza. Anais... Fortaleza: Rede Pró-Unidades de Conservação, 2002.

MINAS GERAIS. Lei n.12.040, de 28 de dezembro de 1995. Dispõe sobre a distribuição da parcela de receita do produto da arrecadação do ICMS pertencente aos municípios. Diário Oficial do Estado de Minas Gerais, Belo Horizonte, dez. 1995.

MINAS GERAIS. Lei n.13.803, de 27 de dezembro de 2000. Dispõe sobre a apuração e distribuição da parcela proveniente da arrecadação do ICMS pertencente aos municípios. Diário Oficial do Estado de Minas Gerais, Belo Horizonte, dez. 2000. 
MINAS GERAIS. Secretaria Estadual do Meio Ambiente e Desenvolvimento Sustentável. ICMS ecológico: o que é, critérios e legislações. Belo Horizonte: SEMAD, 2005a. Disponível em: <http:// www.semad.mg.gov.br/icms_oque.asp>. Acesso em: 10 nov. 2010.

MINAS GERAIS.Secretaria Estadual do Meio Ambiente e Desenvolvimento Sustentável. Resolução n. 318, de 15 de fevereiro de 2005. Disciplina o cadastramento das unidades de conservação da natureza e a divulgação periódica das informações básicas a elas pertinentes. Belo Horizonte: SEMAD, 2005c.

MINAS GERAIS. Lei Estadual 18.030 de 12 de janeiro de 2009. Dispõe sobre alterações sobre a forma de distribuição do ICMS Ecológico aos municípios de 2009. Diário Oficial do Estado de Minas Gerais, Belo Horizonte, jan. 2009.

MINAS GERAIS. Conselho de Meio Ambiente. Deliberação Normativa COPAM nº 86 de 17 de julho de 2005. Belo Horizonte: SEMAD, 2005c.

RIBEIRO,V.D. O ICMS Ecológico como instrumento de Política Florestal. Monografia, Instituto de Florestas UFRRJ, Seropédica, 2008.

TNC-The NatureConservance. O ICMS ecológico no Brasil. Disponível em <http:// www.tnc.org.br/icmsecologico>. Acesso em: 10 nov. 2010. 